\section{NEW TEETH IN A DAY}

At United Smile Centres patients leave happy and transformed with the unique 'Permanent Teeth in a Day' treatment.

This technique is a proven alternative to traditional dentures and is also ideal for patients previously considered unsuitable for standard dental implants.

By using the dense bone that remains in the front part of the jaw, United Smile's expert dentists can place implants at an angle, avoiding the sinus cavities in the upper jaw and the nerve canal in the lower jaw. There is no need for bone grafting and lengthy healing times and patients can leave the United Smile Centre with a fully fixed arch of non-removable teeth on as few as four implants all in the same day.

Your patients will thank you for referring them to United Smile Centres; within just one day they can look forward to a minimum recovery time with very little pain and discomfort. The treatment helps prevent further bone loss, restores the ability to eat all types of food; replaces dentures for a lifetime; requires easy maintenance through oral hygiene; renews youthful appearance; and improves self confidence and quality of life.

For more information call 08008494959 or visit www.unitedsmilecentres.co.uk.

\section{SMART DENTAL MICROMOTOR}

Thanks to Smart Logic technology, the Optima MX2 INT dental micromotor control unit offers ultimate regulation of the power required, linearly and without vibration, ensuring you are perfectly in control of the speed, torque and reversal at all times. With Easy-Nav philosophy the Optima MX2 INT is incredibly intuitive and adapts to most dental chairs. Being particularly versatile the control unit offers ten pre-programmed modes for your main restorative work and ten sequences for your endodontic work, all at the quick press of a button.

\title{
EQUIPMENT PIONEERS MARK HALF CENTURY
}

2012 marks KaVo Dental's 50th anniversary in the UK market, having launched in 1962. Over the last 50 years, KaVo have pioneered the world's first fibre optics, launched the industry's first rental scheme, sold over 200,000 turbines and sold around 150,000 dental chairs.

KaVo was founded in Germany in 1909 and soon focused on designing and producing the very best dental equipment. Back in 1965, KaVo redefined the industry with the 1025 unit, the world's first dental chair to allow the patient to lie down.

\section{TRY BEFORE YOU BUY}

The Varios 970 ultrasonic scaler from NSK is a stylish, compact unit with an extensive range of over 70 tips, making it the ideal choice to treat a wide variety of conditions including perio, endo and scaling, or where minimal intervention is required. The newly developed iPiezo engine provides stable vibration and power with excellent illumination of the treatment area guaranteed by twin LEDs. Each Varios 970 is supplied with a twin LED handpiece, three tips, three tip wrenches and an autoclavable container.

Those looking for a more compact unit would benefit from either the versatile Varios 570, or the Varios 370, specifically designed as a portable control unit for easy installation into any dental unit. Additionally, the
Ergonomics are still a key part of the business's focus today.

KaVo's SonicFill with Kerr was the world's first handpiece with an automated composite delivery system.

$\mathrm{KaVo}$ UK sell their range of high quality equipment, imaging and handpieces across the UK via their dealer partners and offer customer service, marketing support and a fully equipped repair facility from their head offices in Amersham, Buckinghamshire.

For more information on KaVo UK, see www.kavo.co.uk, email info@kavo. com or telephone 01494733000.
Varios 170 is available as a built-in unit, where all its functions can be controlled via NSK's new MultiPad.

For those looking for effective air scaling NSK's new Ti-Max S970 offers an adjustable power range, delivering effective and powerful scaling in a solid titanium body which provides a steady, comfortable grip.

NSK's Prophy-Mate neo is a proven air-driven tooth polishing system that makes cleaning and polishing procedures easier and more effective. Patients will love the feel of their teeth after the FLASH pearl polishing powder has gently removed superficial stains and calculus.

NSK offer a no-obligation ten day 'try before you buy' offer with the Varios 970 and Prophy-Mate neo. Call NSK on 0800 6341909 or visit www.nsk-uk.com.
This Swiss made control unit has been developed with your endodontic work in mind. The Optima MX2 INT has been designed to keep the risk of NiTi instrument breakage to an

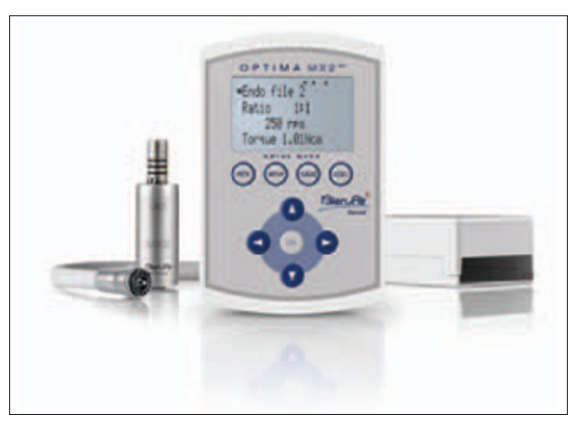

absolute minimum with the precise auto reverse function.

Optima MX 2 INT drives the new LED MX 2 Micromotor. MX 2 is shorter and lighter than its predecessor and with its shortened nose accepts BienAir's new Micro-Series contra-angles, as well as standard contra-angles. The combination of both the MX 2 and Micro-series is a reduction in the overall length of 30\% and a weight reduction of $23 \%$.

For further information contact Bien-Air on 01293550200 or visit www.bienair.com. 